\title{
ARTICLE
}

\section{AFFl and AFF4 differentially regulate the osteogenic differentiation of human MSCs}

Chen-chen Zhou ${ }^{1 *}$, Qiu-chan Xiong ${ }^{1 *}$, Xin-xing $\mathrm{Zhu}^{2}$, Wen $\mathrm{Du}^{1}$, Peng Deng ${ }^{1,3}$, Xiao-bing Li ${ }^{1,4}$, Yi-zhou Jiang $^{2}$, Shu-juan Zou ${ }^{1,4}$, Cun-yu Wang ${ }^{3}$ and Quan Yuan ${ }^{1,5}$

AFF1 and AFF4 belong to the AFF (AF4/FMR2) family of proteins, which function as scaffolding proteins linking two different transcription elongation factors, positive elongation factor $b$ (P-TEFb) and ELL1/2, in super elongation complexes (SECs). Both AFF1 and AFF4 regulate gene transcription through elongation and chromatin remodeling. However, their function in the osteogenic differentiation of mesenchymal stem cells (MSCs) is unknown. In this study, we show that small interfering RNA (siRNA)-mediated depletion of AFF1 in human MSCs leads to increased alkaline phosphatase (ALP) activity, enhanced mineralization and upregulated expression of osteogenic-related genes. On the contrary, depletion of AFF4 significantly inhibits the osteogenic potential of MSCs. In addition, we confirm that overexpression of AFF1 and AFF4 differentially affects osteogenic differentiation in vitro and MSC-mediated bone formation in vivo. Mechanistically, we find that AFF1 regulates the expression of DKK1 via binding to its promoter region. Depletion of DKK1 in HA-AFF1-overexpressing MSCs abrogates the impairment of osteogenic differentiation. Moreover, we detect that AFF4 is enriched in the promoter region of ID1. AFF4 knockdown blunts the BRE luciferase activity, SP7 expression and ALP activity induced by BMP2 treatment. In conclusion, our data indicate that AFF1 and AFF4 differentially regulate the osteogenic differentiation of human MSCs.

Bone Research (2017) 5, 17044; doi:10.1038/boneres.2017.44; published online: 26 September 2017

\section{INTRODUCTION}

Mesenchymal stem cells (MSCs) are pluripotent stem cells that can differentiate into osteoblastic, chondrogenic, and adipogenic lineages. ${ }^{1-2}$ The bone marrow is the main source of stem cells for mesenchymal tissue.' Apart from that, MSCs can also be found in many other parts of the human body, including adipose tissue, chorionic villi of the placenta, amniotic fluid, peripheral blood, fetal liver, lung, and teeth. ${ }^{3-6}$

The osteogenic differentiation of MSCs is a complex process involving numerous signal molecules, including key transcription factors such as runt-related transcription factor
2 (Runx2) and Osterix, as well as various hormones. ${ }^{7-11}$ In addition, the Wnt/ $\beta$-catenin, bone morphogenetic protein (BMP) and transforming growth factor- $\beta$ (TGF- $\beta$ ) signaling pathways are indispensable during the osteogenic process. ${ }^{12-15}$ Recently, accumulating evidence has shown that epigenetic regulation plays a pivotal role in the osteogenic differentiation of MSCs. ${ }^{16-18}$ DNA methylation and histone modifications are the major mammalian epigenetic mechanisms involved in the progression from MSCs into terminally differentiated cells. ${ }^{16,19-21}$ For example, the expression level of histone deacetylase 1 (HDAC1) is decreased during osteoblast differentiation. ${ }^{21}$

\footnotetext{
'State Key Laboratory of Oral Diseases \& National Clinical Research Center for Oral Diseases, West China Hospital of Stomatology, Sichuan University, Chengdu, China; ${ }^{2}$ Institute for Advanced Study, Shenzhen University, Shenzhen, China; ${ }^{3}$ Laboratory of Molecular Signaling, Division of Oral Biology and Medicine, School of Dentistry and Broad Stem Cell Research Center, UCLA, Los Angeles, CA, USA; ${ }^{4}$ Department of Orthodontics, West China Hospital of Stomatology, Sichuan University, Chengdu, China and ${ }^{5}$ Department of Oral Implantology, West China Hospital of Stomatology, Sichuan University, Chengdu, China

Correspondence: Quan Yuan (yuanquan@scu.edu.cn) or Cun-yu Wang (cunywang@ucla.edu)

*These authors contribute equally to this work.

Received: 16 April 2017; Revised: 18 June 2017; Accepted: 18 June 2017
} 
Both AFF1 and AFF4 belong to the AFF (AF4/FMR2) family and regulate gene transcription epigenetically through elongation and chromatin remodeling. ${ }^{22-24}$ They share three conserved domains: an N-terminal homology domain, an AF4/lymphoid nuclear protein domain, and a C-terminal homology domain. ${ }^{25}$ Both function as scaffolding proteins linking two different transcription elongation factors, positive elongation factor $b$ (P-TEFb) and ELL1/2, in super elongation complexes (SECs). ${ }^{26-27}$ Studies have shown that AFFl and AFF4 are associated with acute lymphoblastic leukemia and FRAXE mental retardation. ${ }^{27-31}$ AFFl promotes CD133 transcription and leukemia cell survival in multiple cancer cell lines. ${ }^{32}$ AFFl and AFF4 also play important roles in HIV transactivation and are closely associated with HIV-1 Tat. $^{33-34}$ However, the role of AFFl and AFF4 in MSC osteogenic differentiation is largely unknown.

Although AFFl and AFF4 are members of the same protein family with common structures and functions, we show that they differentially regulate the osteogenic differentiation of human MSCs in vitro and MSC-mediated bone formation in vivo. Mechanically, AFFl controls the transcription of Dickkopf-related protein 1 (DKK1), while AFF4 is required for DNA-binding protein inhibitor IDI transcription and BMP2-induced responses.

\section{MATERIALS AND METHODS}

Cell culture

Human bone marrow-derived mesenchymal stem cells (MSCS) were obtained from the American Type Culture Collection (ATCC, Manassas, VA, USA). The cells were cultured in Dulbecco's modified Eagle's medium (DMEM) supplemented with 10\% fetal bovine serum (FBS, Gibco, Waltham, MA, USA), $100 \mathrm{U} \cdot \mathrm{mL}^{-1}$ penicillin and $100 \mathrm{mg} \cdot \mathrm{mL}^{-1}$ streptomycin (Gibco) at $37^{\circ} \mathrm{C}$ in a humidified atmosphere of $5 \% \mathrm{CO}_{2}$. To induce osteogenic differentiation, the cells were treated with osteogenic medium containing $50 \mu \mathrm{mol} \cdot \mathrm{L}^{-1}$ ascorbic acid, $10 \mathrm{mmol} \cdot \mathrm{L}^{-1} \beta$ glycerophosphate, and $10 \mathrm{nmol} \cdot \mathrm{L}^{-1}$ dexamethasone (all from Sigma, St. Louis, MO, USA). ${ }^{35}$ All experimental protocols and procedures were approved by the State Key Laboratory of Oral Diseases, West China Hospital of Stomatology, Sichuan University.

Gene knockdown and overexpression

We obtained targeted and control small interfering RNAs (siRNAs) from Santa Cruz Biotechnology (Santa Cruz, Dallas, TX, USA), and transfections were performed using Lipofectamine RNAiMAX reagent (Invitrogen) according to the manufacturer's instructions. The knockdown efficiency was verified by quantitative reverse transcription PCR (RT-PCR) and western blot 2 days after transfection.
For overexpression, lentiviruses expressing HA-AFFl, HAAFF4 or empty vectors were purchased from GeneCopoeia (Guangzhou, China). MSCs were infected with these viruses in the presence of Polybrene (Sigma) for $24 \mathrm{~h}$ and selected by treatment with $1 \mu \mathrm{g} \cdot \mathrm{mL}^{-1}$ puromycin (Sigma). The infection efficiency in the selected stable cells was confirmed by quantitative reverse transcription PCR (RT-PCR) and western blot.

RNA isolation and quantitative RT-PCR

Total RNA was isolated using TRIzol reagent (Invitrogen, Waltham, MA, USA) according to the manufacturer's instructions. CDNA was prepared from $1 \mu \mathrm{g}$ aliquots of RNA using a Primescript RT reagent kit (Takara, Dalian, China). Quantitative real-time PCR was performed using SYBR Premix Ex Taq (Takara) with an ABI 7500 real-time PCR system (Applied Biosystems, Foster City, CA, USA). ${ }^{36}$ Relative expression levels were calculated using the $2^{-\Delta \Delta C \dagger}$ method by normalization to the expression of the Gadph housekeeping gene and were presented as the fold increase relative to the control. ${ }^{9}$

Western blot

MSCs were lysed on ice in lysis buffer containing $50 \mathrm{mmol} \cdot \mathrm{L}^{-1}$ Tris- $\mathrm{HCl}, \quad 150 \mathrm{mmol} \cdot \mathrm{L}^{-1} \mathrm{NaCl}, 1 \mathrm{mmol} \cdot \mathrm{L}^{-1}$ EDTA, $1 \%$ Nonidet P-40, and a protease inhibitor cocktail (Roche, Indianapolis, IN, USA). The cells were then centrifuged at $18000 \times g$ for $15 \mathrm{~min}$ at $4^{\circ} \mathrm{C}$ to remove the cell debris. The supernatants were heated at $95^{\circ} \mathrm{C}$ for $5 \mathrm{~min}$ in sample buffer containing 2\% SDS and 1\% 2-mercaptoethanol, separated on 10\% SDS-polyacrylamide gels, and transferred to PVDF membranes using a wet transfer apparatus (Bio-Rad, Hercules, CA, USA). The membranes were blocked with $5 \%$ milk for $1 \mathrm{~h}$ and then incubated with rabbit anti-AFF1 (Bethyl, Montgomery, TX, USA, 1:1 000), rabbit anti-AFF4 (Abcam, Cambridge, MA, USA, 1:1 000), rabbit anti-DKK1 (Cell Signaling, Danvers, MA, USA, 1:1 000), rabbit anti-ID1 (Abcam, 1:1000), or mouse anti- $\alpha$-tubulin (Sigma, 1:5000) overnight, followed by a horseradish peroxidase-conjugated anti-rabbit or anti-mouse lgG (Jackson ImmunoResearch, West Grove, PA, USA). The antibodyantigen complexes were visualized with SuperSignal reagents (Pierce, Rockford, IL, USA).

Cell proliferation assay

The proliferation of MSCs was analyzed using Cell Proliferation Reagent WST-1 (Roche). Briefly, $10 \mu \mathrm{L}$ of reagent was added to each well, including five wells containing only medium for background subtraction. After a 1-hour incubation at $37^{\circ} \mathrm{C}$, the absorbance at $450 \mathrm{~nm}$ was measured using a Varioskan Flash microplate reader (Thermo Scientific, Waltham, MA, USA). 


\section{ALP staining and activity}

For ALP staining, cells were seeded in 24-well culture plates. After reaching confluence, the cells were incubated with osteogenic differentiation medium for 7 days. The cells were then fixed in $70 \%$ ethanol and incubated with a staining solution of $0.25 \%$ naphthol AS-BI phosphate and $0.75 \%$ Fast Blue BB dissolved in $0.1 \mathrm{~mol} \cdot \mathrm{L}^{-1}$ Tris buffer $(\mathrm{pH}$ 9.3). The ALP activity was quantified using a commercial kit according to the manufacturer's protocol (Cell Biolab, San Diego, CA, USA) and normalized to the total protein levels. ${ }^{36}$

\section{Alizarin red staining}

MSCs were cultured in osteogenic medium for 2-3 weeks, fixed with $10 \%$ neutral formalin for $5 \mathrm{~min}$, and stained with $2 \%$ Alizarin red S (pH 4.2, Sigma) for 10 min. ${ }^{37}$ Mineralized bone nodules stained with Alizarin red were destained with $10 \%$ cetylpyridinium chloride in $10 \mathrm{mmol} \cdot \mathrm{L}^{-1}$ sodium phosphate (pH 7.0), and the calcium concentration was determined by measuring the absorbance at $562 \mathrm{~nm} .^{36}$
MSC transplantation

Three-month-old immunocompromised beige mice were obtained from the Experimental Animal Center of the university and housed in pathogen-free facilities under a 12-h light and 12-h dark cycle. All procedures were conducted in accordance with The Guidelines for the Care and Use of Laboratory Animals of the State Key Laboratory of Oral Diseases, West China Hospital of Stomatology, Sichuan University. Approximately $5 \times 10^{6}$ cells were mixed with $60 \mathrm{mg}$ of pure phase beta-tricalcium phosphate particles (SynthoGraft, Bicon, Boston, MA) and then transplanted subcutaneously under the dorsal surface as previously described. ${ }^{36}$ The samples were collected 3 and 6 weeks after transplantation and decalcified with $10 \%$ EDTA. Paraffin sections were generated and stained with hematoxylin and eosin.

\section{Chromatin immunoprecipitation assay}

The chromatin immunoprecipitation (ChIP) assay was performed using a Simple ChIP Assay Kit (Cell Signaling a

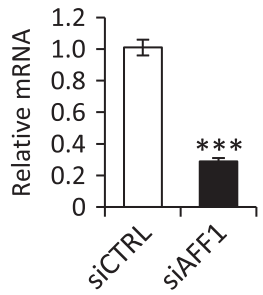

d

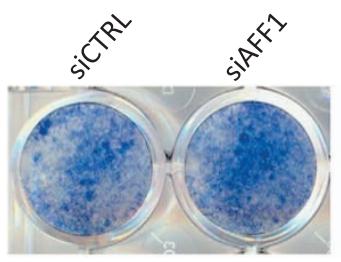

b

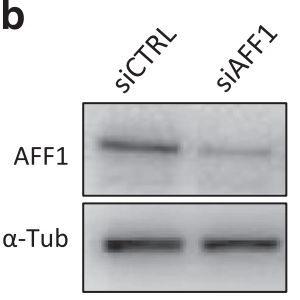

C

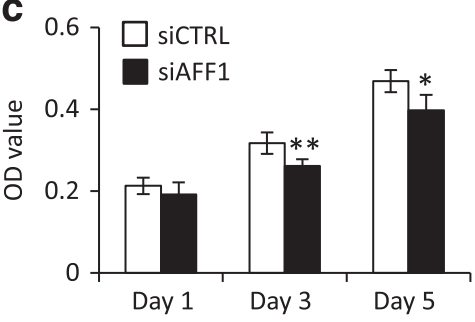

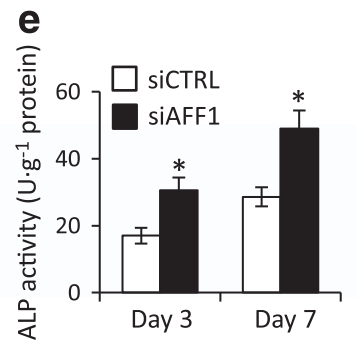

f

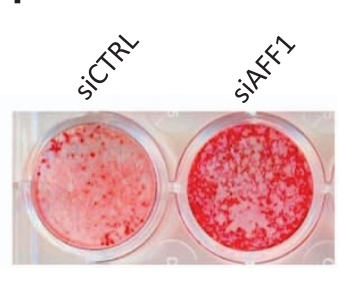

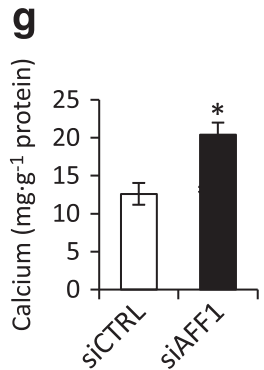

h

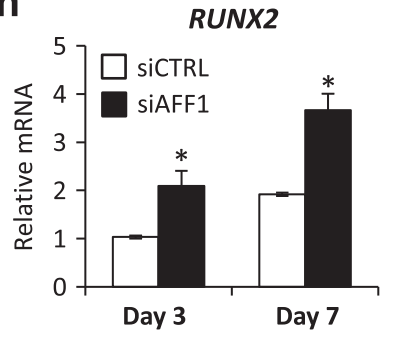

i

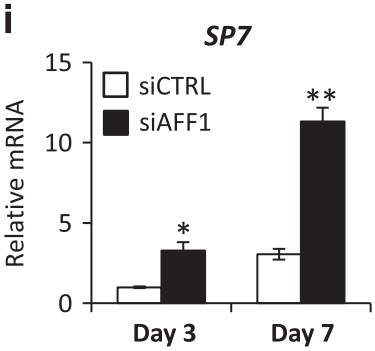

j

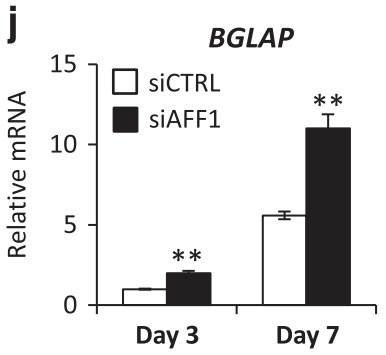

Figure 1. Depletion of AFF1 improves osteogenic differentiation. (a) Real-time RT-PCR shows successful knockdown of $A F F 1$. $n=3$. ${ }^{* * *} P<0.001$. (b) Western blot analysis of AFF1. (c) Depletion of AFF1 inhibits the proliferation of MSCs. $n=5 .{ }^{*} P<0.05$ and ${ }^{* *} P<0.01$. (d) Representative images of alkaline phosphatase (ALP) staining. Depletion of AFF1 leads to more intense staining. (e) Quantitative analyses of ALP activity. $n=5$. ${ }^{*} P<0.05$. (f) Representative images of Alizarin red S (ARS) staining of MSCs. (g) Quantitative analyses of calcium mineralization. $n=5$. ${ }^{*} P<0.05$. (h-j) Realtime RT-PCR reveals elevated mRNA expression of RUNX2, SP7 and BGLAP. $n=3 .{ }^{*} P<0.05$ and ${ }^{* *} P<0.01$. 

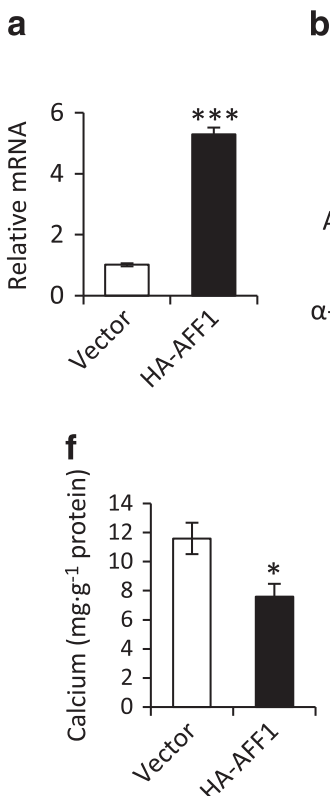

b

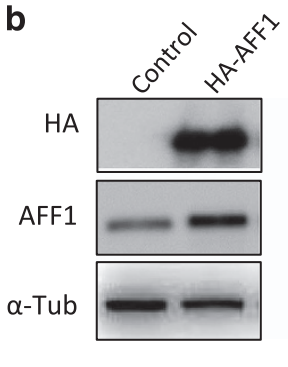

g

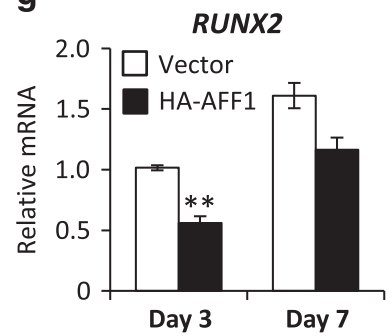

c

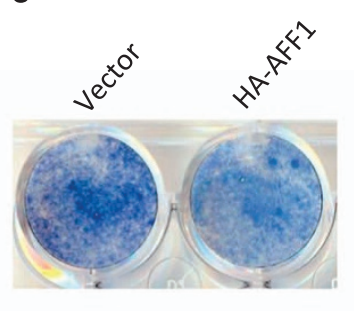

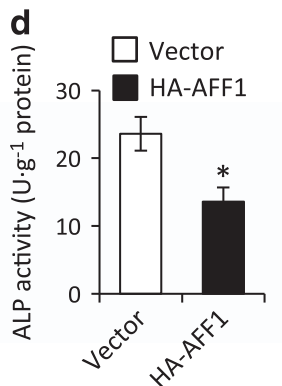

e

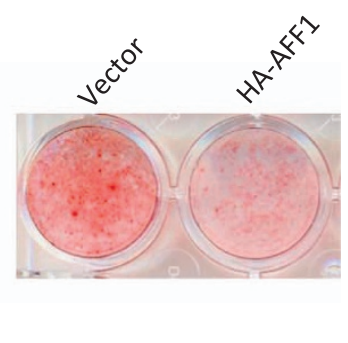

h

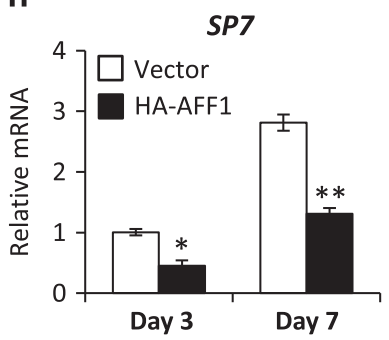

i

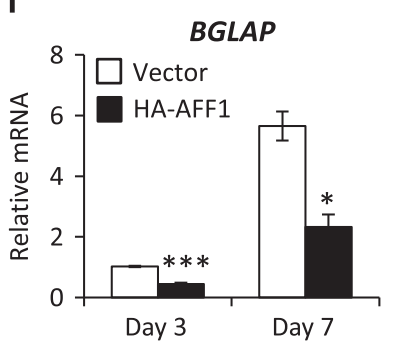

Figure 2. Overexpression of AFF1 impairs osteogenic differentiation. (a) Real-time RT-PCR shows that the mRNA level of AFF1 is significantly increased. $n=3$. ${ }^{* * *} P<0.001$. (b) Western blot analysis of AFF1. (c) Representative images of ALP staining. Overexpression of AFF1 decreases the intensity of the staining. (d) Quantitative analyses of ALP activity. $n=5 .{ }^{*} P<0.05$. (e) Representative images of ARS staining of MSCs. (f) Quantitative analyses of calcium mineralization. $n=5 .{ }^{*} P<0.001$. (g-i) Real-time RT-PCR reveals decreased mRNA expression of RUNX2, SP7 and BGLAP. $n=3$. ${ }^{*} P<0.05,{ }^{* *} P<0.01$ and ${ }^{* * *} P<0.001$.

Technology) according to the manufacturer's protocol with an antibody against AFF1 (Bethyl), AFF4 (Abcam) or the control normal rabbit IgG (Santa Cruz Biotechnology). After dissociating the DNA-protein complexes, the pulled-down DNA and input DNA were subject to quantitative RT-PCR analysis with primers designed to amplify target promoter regions. The results were expressed as the percentage of input DNA.

\section{Luciferase assay}

MSCs were transfected with AFF4 or control siRNA together with $100 \mathrm{ng}$ of BRE luciferase (\#45126 Addgene, Cambridge, MA, USA) and 50 ng of CMV- $\beta$-galactosidase constructs using Lipofectamine 2000 transfection reagent (Invitrogen). After approximately $24 \mathrm{~h}$, the cells were starved in serum-free medium overnight followed by treatment with $100 \mathrm{ng} \cdot \mathrm{mL}^{-1}$ BMP2 for $6 \mathrm{~h}$. The luciferase and $\beta$-galactosidase activities of total cell lysates were determined using Luc-Screen and Galacto-Star kits (Applied Biosystems).

\section{Statistical analysis}

All values are presented as the mean \pm s.e. A two-tailed Student's t-test and one-way analysis of variance (ANOVA) followed by Tukey's test were used for single comparisons and multiple comparisons to assess the statistical significance of the differences among each pair of data sets. A $P$-value $<0.05$ was considered to be statistically significant.

\section{RESULTS}

Depletion of AFF1 improves the osteogenic differentiation of MSCs

To investigate the role of AFF1 in osteogenic differentiation, we first depleted AFF1 in human MSCs using siRNA. The knockdown efficiency was confirmed by RT-PCR and western blot (Figure la and b). AFF1 depletion decreased the proliferation of MSCs (Figure 1c). After osteogenic induction for 7 days, we found that siRNA-mediated depletion of AFF1 led to more intense staining of alkaline phosphatase (ALP), an early marker of osteoblastic differentiation (Figure 1d). This observation was confirmed by quantitatively measuring ALP activity on days 3 and 7 (Figure 1e). We also assessed extracellular matrix (ECM) mineralization by Alizarin red S (ARS) staining. As shown in Figure If and $g$, mineralization was significantly increased after AFFl depletion. In addition, AFFl knockdown upregulated the expression of osteogenic-related genes, such as Runx2, Osterix (SP7), and Osteocalcin (BGLAP) (Figure 1h-j).

Overexpression of AFF1 impairs osteoblastic differentiation To confirm the role of AFF1 in osteoblastic differentiation, we investigated the effect of ectopic overexpression of AFF1 on osteoblastic differentiation. Human MSCs were stably transduced with lentiviral particles expressing HAAFFl (Figure $2 a$ and $b$ ). We found that overexpression of AFFl decreased the ALP activity and mineralization of MSCs (Figure 2c-f). In addition, the expression of 
a

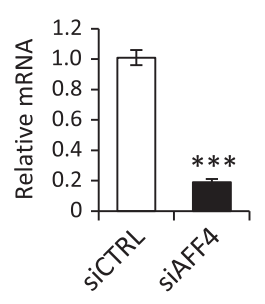

b

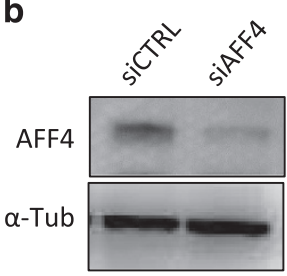

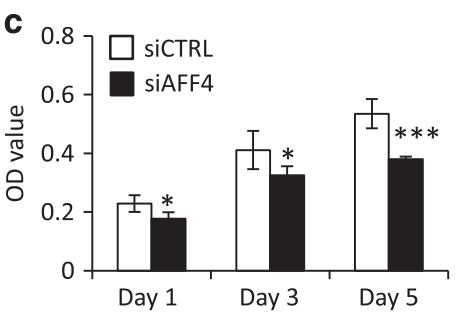

C

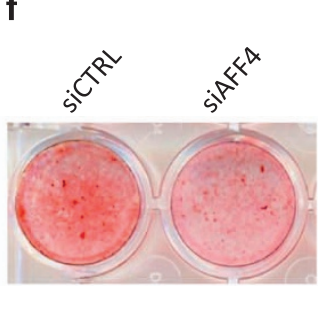

g

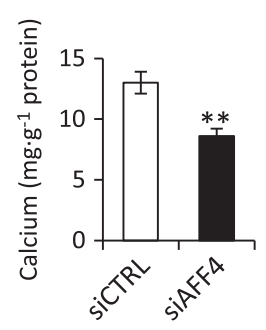

h RUNX2

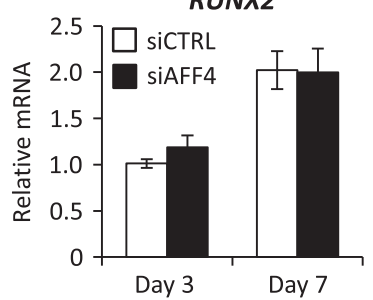

i
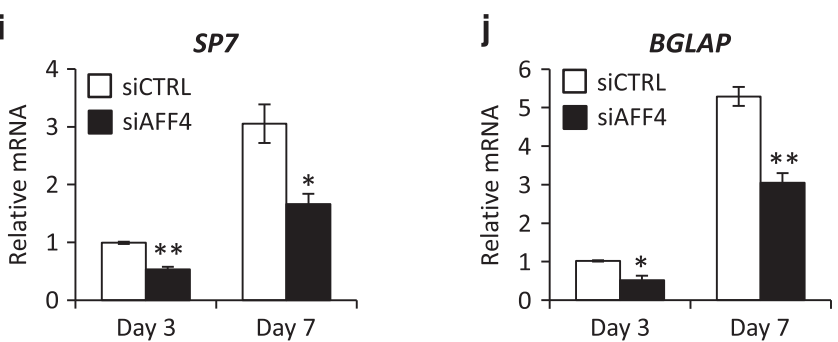

Figure 3. Depletion of AFF4 decreases osteogenic differentiation. (a) Real-time RT-PCR shows successful knockdown of $A F F 4$. $n=3$. ${ }^{* * *} P<0.001$. (b) Western blot analysis of AFF4. (c) Depletion of AFF4 reduces the proliferation of MSCs. $n=5$. ${ }^{*} P<0.05$ and ${ }^{* * *} P<0.001$ (d) Representative images of ALP staining. Depletion of AFF4 decreases the intensity of the staining. (e) Quantitative analyses of ALP activity. $n=5$. ${ }^{*} P<0.05$. (f) Representative images of ARS staining of MSCs. (g) Quantitative analyses of calcium mineralization. $n=5$. ${ }^{* *} P<0.01$. (h-j) Real-time RT-PCR reveals reduced mRNA expression of $S P 7$ and BGLAP. $n=3$. ${ }^{*} P<0.05$ and ${ }^{* *} P<0.01$.

osteogenic-related genes, such as Runx2, SP7, and BGLAP, was significantly repressed in AFFl-depleted cells subjected to osteogenic induction for 5 days (Figure $2 \mathrm{~g}-\mathrm{i}$ ).

Depletion of AFF4 decreases osteogenic differentiation As both AFF4 and AFFl are key members of the AFF family and share highly conserved functional domains involved in gene transcription regulation, including conserved $\mathrm{N}$ - and C-terminal domains, a serine-rich transactivation domain, and an ALF homology region, 25,38 we sought to verify whether AFF4 plays a similar role as AFF1 in regulating the osteogenic differentiation of MSCs. AFF4 was knocked down in MSCs using siRNA (Figure $3 a$ and b), resulting in reduced MSC proliferation (Figure 3c). Surprisingly, depletion of AFF4 significantly reduced the alkaline phosphatase (ALP) activity and extracellular matrix mineralization, indicating that it had an opposite effect on osteogenic differentiation compared with AFFl (Figure 3d-g). In addition, knockdown of AFF4 inhibited the expression of osteogenic-related genes, such as RUnx2, SP7, and BGLAP (Figure 3h-j).
Overexpression of AFF4 enhances the osteoblastic differentiation of MSCs

To investigate the effects of ectopic overexpression of AFF4 on osteoblastic differentiation, human MSCs were stably transduced with lentiviral particles expressing HA-AFF4 (Figure $4 \mathrm{a}$ and b). As expected, the ALP activity and mineralization of MSCS were markedly enhanced after AFF4 overexpression (Figure 4C-f). Moreover, an RT-PCR assay showed that the expression of the abovementioned osteogenic-related genes was significantly elevated after AFF4 overexpression and 5 days of osteogenic induction (Figure 4h-i).

AFF1 and AFF4 differentially regulate MSC-mediated bone formation in vivo

To verify our findings in vitro, we examined whether the overexpression of AFF1 and AFF4 differentially affected MSC-mediated bone formation in vivo. To this end, we subcutaneously implanted MSCs stably overexpressing HAAFF1 or HA-AFF 4 with $\beta$-TCP scaffolds into immunocompromised mice. After 3 weeks, H\&E staining showed that there was very little newly generated bone, while many $\beta$-TCP 

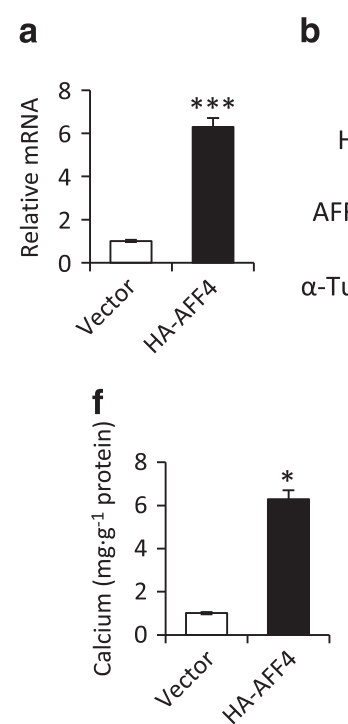

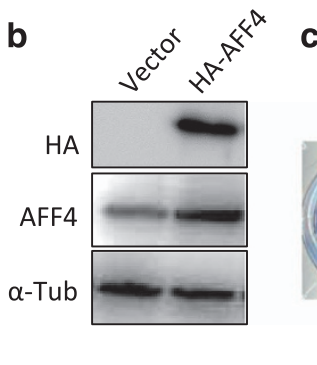

g

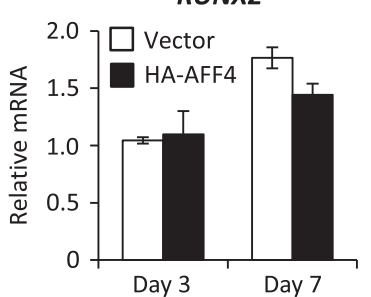

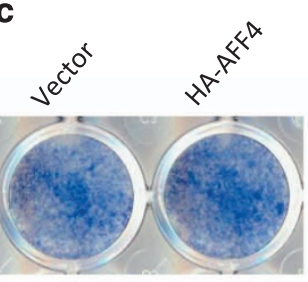

d

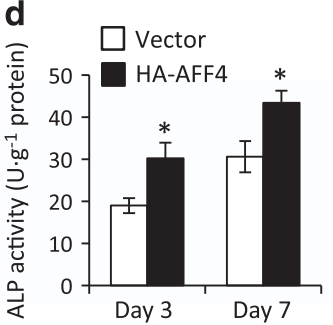

e

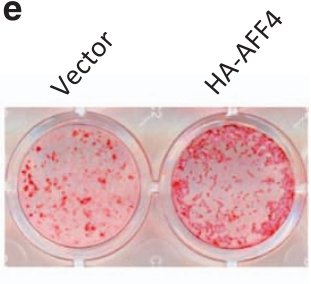

h

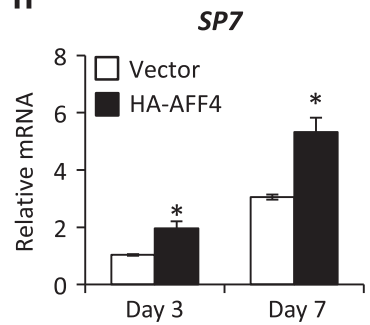

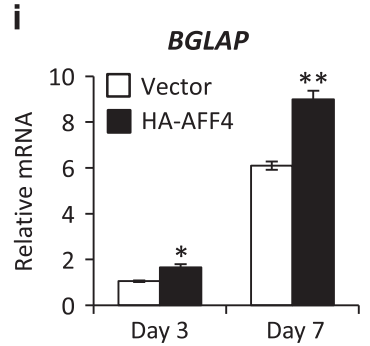

Figure 4. Overexpression of AFF4 enhances osteogenic differentiation. (a) Real-time RT-PCR shows that the mRNA level of AFF4 is significantly increased. $n=3$. ${ }^{* * *} P<0.001$. (b) Western blot analysis of AFF4. (c) Representative image of ALP staining. Overexpression of AFF4 leads to more intense staining. (d) Quantitative analyses of ALP activity. $n=5$. ${ }^{*} P<0.05$. (e) Representative images of ARS staining of MSCs. (f) Quantitative analyses of calcium mineralization. $n=5 .{ }^{*} P<0.001$. (g-i) Real-time RT-PCR reveals increased mRNA expression of $S P 7$ and $B G L A P . n=3$. ${ }^{*} P<0.05$ and ${ }^{* *} P<0.01$.

a

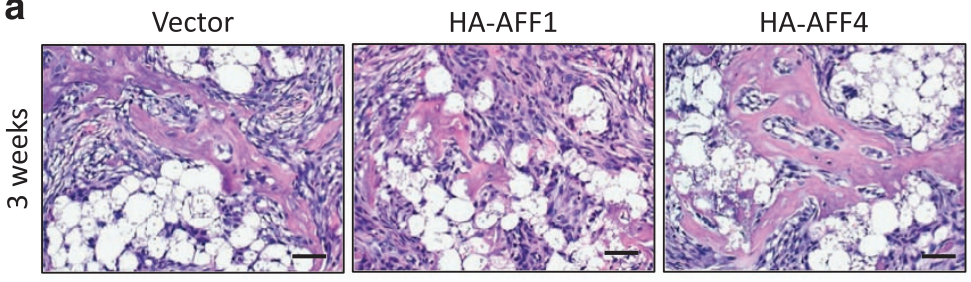

C

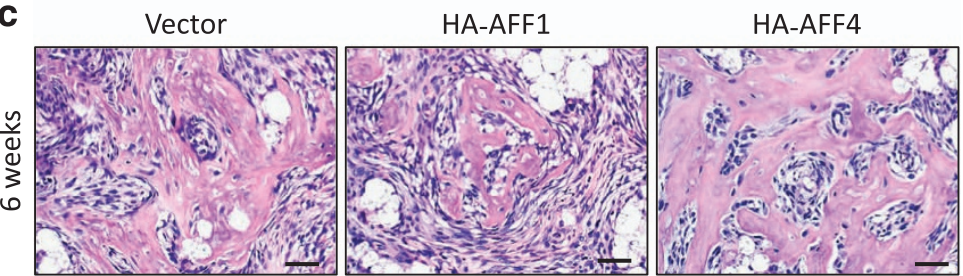

b

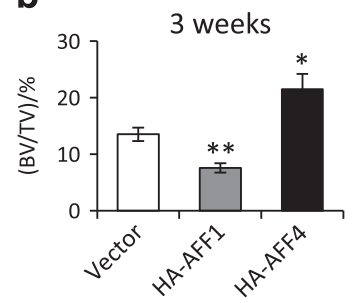

d

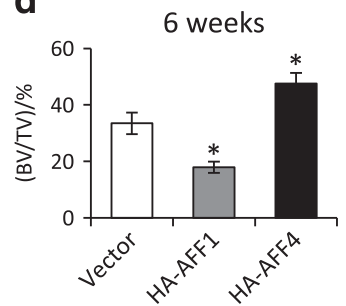

Figure 5. AFF1 and AFF4 differentially regulate bone formation in vivo. (a) Representative images of H\&E staining of ectopic bone formation at 3 weeks. There is very little newly generated bone, while many $\beta$-TCP particles (the white bubble-like spots) remain. (b) Quantitative analyses of bone volume versus total tissue volume (BV/TV) at 3 weeks. $n=5$. ${ }^{*} P<0.05$ and ${ }^{* *} P<0.01$. (c) Representative images of H\&E staining at 6 weeks. Cells overexpressing HA-AFF1 form less bone tissue, while those overexpressing HA-AFF4 exhibit more bone formation. (d) BV/TV at 6 weeks. $n=5 .{ }^{*} P<0.05$

particles remained (Figure 5a). However, the bone volume in the HA-AFF4 group was significantly higher than that in the control and HA-AFFl groups (Figure $5 a$ and b). At 6 weeks, much more bone was observed in all three groups compared with that at 3 weeks. Mice implanted with AFF1overexpressing MSCs showed much less bone tissue (Figure $5 \mathrm{c}$ and d). By contrast, increased bone formation was observed in mice implanted with AFF4-overexpressing MSCs (Figure $5 c$ and d), and bone marrow was observed.

\section{AFF1 controls DKK1 transcription}

DKK I is an antagonistic inhibitor of the Wnt/ $\beta$-catenin signaling pathway, which plays a critical role in skeletal 


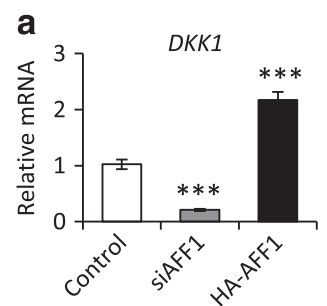

d

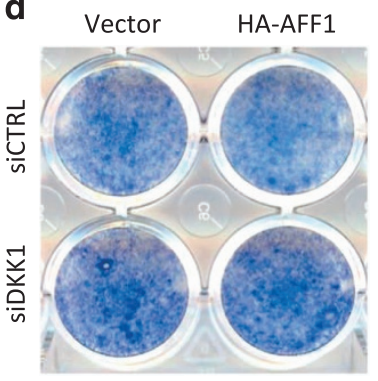

f

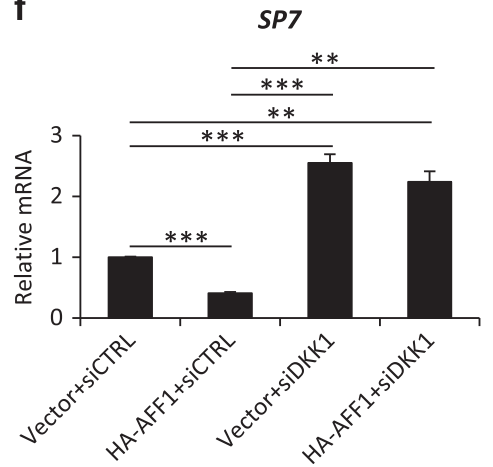

b

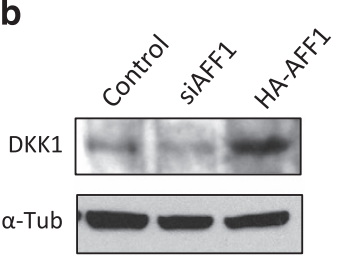

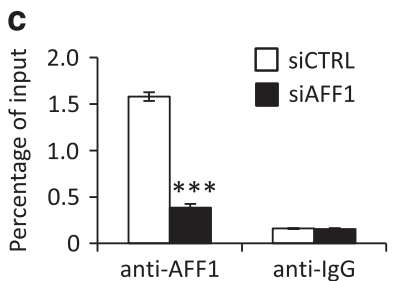

e

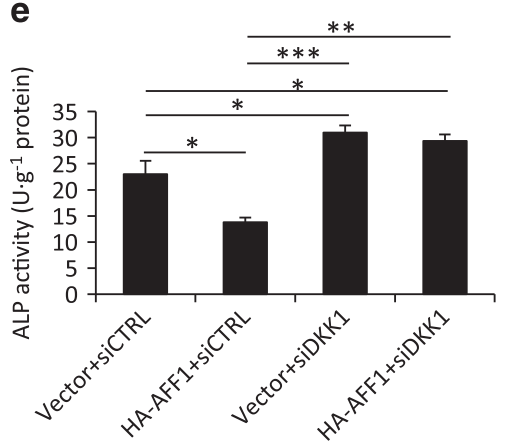

g

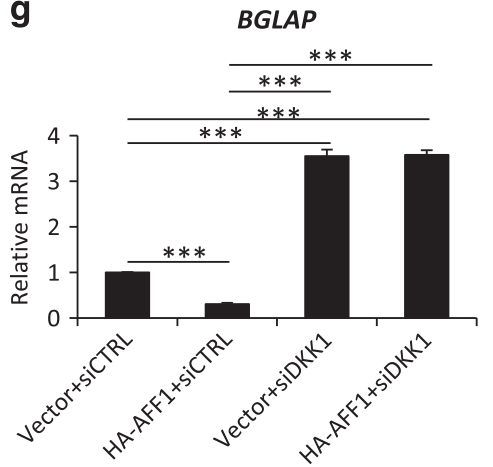

Figure 6. AFF1 controls DKK1 transcription. (a) Real-time RT-PCR shows altered expression of DKK1. $n=3$. ${ }^{* * *} P<0.001$. (b) Western blot analysis of DKK1. (c) A ChIP assay for AFF1 shows that it binds to the promoter region of DKK1. $n=4 .{ }^{* * *} P<0.001$. (d) Representative images of ALP staining. (e) Quantitative analyses of ALP activity. $n=5{ }^{*} P<0.05,{ }^{* *} P<0.01$ and ${ }^{* * *} P<0.001$. (f, g) Real-time RT-PCR of SP7 and $B G L A P$. $n=3$. ${ }^{* *} P<0.01$ and ${ }^{* * *} P<0.001$.

development and osteogenesis. ${ }^{14,39}$ Therefore, we want to know whether AFFl, as an elongation factor, could mediate DKK1 gene transcription. Thus, we examined the expression of DKK1 after AFF1 depletion in MSCs. We found that depletion of AFF1 in MSCs significantly reduced the mRNA and protein levels of DKK1 (Figure 6a and b). Conversely, overexpression of AFF1 increased the expression of DKK I (Figure 6a and b). In addition, we performed an anti-AFFl ChIP assay, which demonstrated that AFF1 bound to the promoter region of DKKI. The ChIP signal for AFFl was almost completely abolished by AFF1 knockdown (Figure 6C). These data suggested that AFF1 might control the osteoblastic differentiation of MSCs by regulating DKK 1 transcription.

To further elucidate the mechanism, we performed rescue experiments by knocking down DKK 1 in stable HAAFFl-expressing MSCs using siRNA. Depletion of DKK 1 significantly abolished the inhibition of ALP activity triggered by AFF1 overexpression (Figure 6d and e) as well as the expression of SP7, a master transcription factor for osteogenic differentiation (Figure 6f), and GBLAP, a marker for mature osteoblasts (Figure 6g).

AFF4 is required for IDI transcription and BMP2-induced responses

The DNA-binding protein inhibitor IDI is a target of the BMP pathway and plays an important role in regulating the differentiation of stem cells. ${ }^{12,40}$ Here, we found that depletion of AFF4 significantly reduced the mRNA level of IDI as well as its protein levels (Figure 7a and b). By contrast, overexpression of AFF4 induced the expression of IDI (Figure 7a and b). We then performed a ChIP assay and observed that AFF4 was enriched in the promoter 

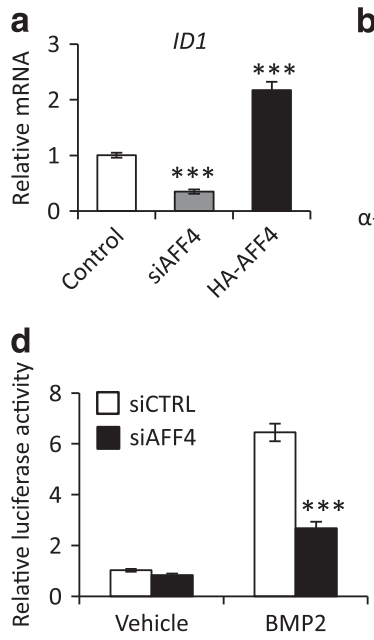

f

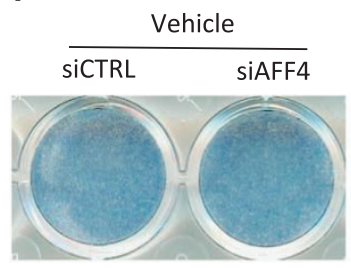

b
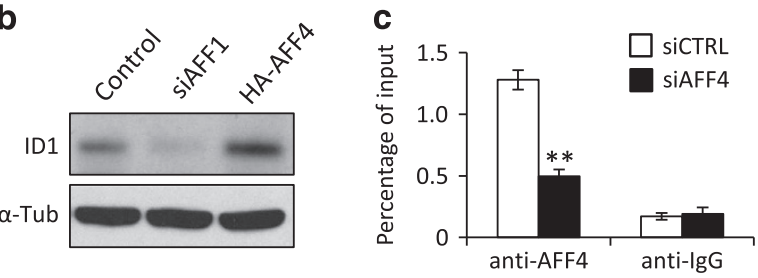

e
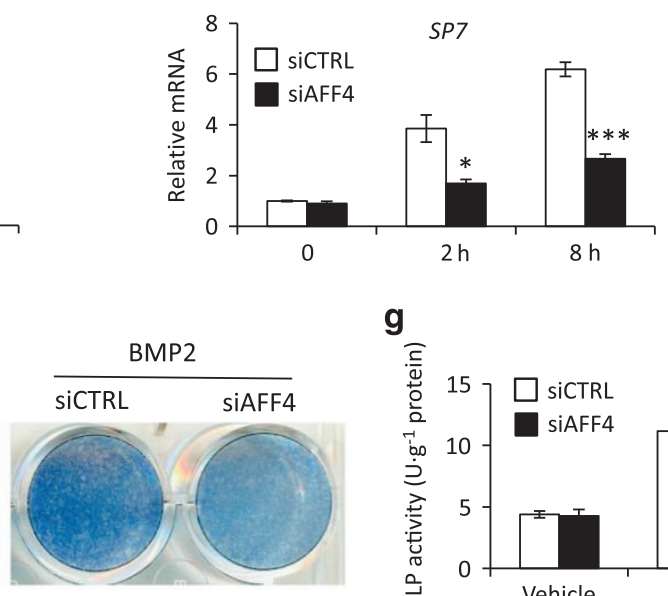

g

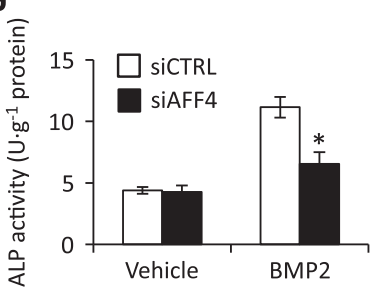

Figure 7. AFF4 is required for ID1 transcription and BMP2-induced responses. (a) Real-time RT-PCR shows altered expression of ID1. $n=3$. ${ }^{* * *} P<0.001$. (b) Western blot analysis of ID1. (c) A ChIP assay for AFF4 shows that it binds to the promoter region of ID1. $n=4$. ${ }^{* *} P<0.01$. (d) Relative luciferase activity after BMP2 $\left(100 \mathrm{ng} \cdot \mathrm{mL}^{-1}\right)$ treatment for $6 \mathrm{~h}$. Depletion of AFF4 blunts the BMP2-induced luciferase activity. $n=4$. ${ }^{* * *} P<0.001$. (e) Real-time RT-PCR indicates that knockdown of AFF4 decreased SP7 expression after BMP2 $\left(100 \mathrm{ng} \cdot \mathrm{mL}^{-1}\right)$ treatment. $n=3$. ${ }^{*} P<0.05$ and ${ }^{* * *} P<0.001$. (f) Representative images of ALP staining. (g) Quantitative analyses of ALP activity. Knockdown of AFF4 decreases the ALP activity induced by BMP2 treatment. $n=5 .{ }^{*} P<0.05$.

region of IDI, suggesting that AFF4 might control osteogenic differentiation via the BMP pathway by regulating IDI transcription (Figure 7c). To this end, we performed a BRE luciferase assay and found that AFF4 depletion markedly restricted BMP2-induced responses (Figure 7d). As BMP2 induces the expression SP7, a master transcription factor for osteogenic differentiation, we examined whether this induction requires AFF4. Quantitative RT-PCR revealed that deletion of AFF4 blunted the expression of SP7 induced by BMP2 treatment (Figure 7e). In addition, knockdown of AFF4 also decreased the ALP activity induced by BMP2 treatment (Figure $7 f$ and $g$ ).

\section{DISCUSSION}

As a set of transcriptional regulators, AFF family proteins play important roles in numerous biological processes, including transcription regulation, chromatin remodeling and leukemia. ${ }^{24,28,41}$ Their relationship with leukemia and functions in HIV transcription has been widely investigated. ${ }^{29-30,33-34}$ Nevertheless, whether they function similarly in cellular differentiation, especially in the osteogenic differentiation of MSCS, remains unknown. In the present study, we show that AFFl depletion enhances the expression of osteogenic-related genes, ALP activity and mineralization. Overexpression of AFF1 impairs osteogenic differentiation and MSC-mediated bone formation in vivo. Conversely, we find that AFF4, another member of the AFF family, has an opposite regulatory function in the expression of osteogenic-related genes, ALP activity and mineralization in vitro as well as in bone formation in vivo. These findings indicate that AFF1 and AFF4 differentially regulate the osteogenic differentiation of MSCs. The expression levels of AFF2 and 3 are too low to be detected in hMSCs (unpublished data).

AFFl and AFF4 share some conserved functional domains in their protein structures. ${ }^{24,41}$ Both of them are reported to form fusion genes with the mixed lineage leukemia (MLL) gene, which is associated with leukemia. ${ }^{32,42}$ Both AFFl and AFF4 act as partners of P-TEFb to regulate transcription elongation. Previous studies have shown that either AFFl or AFF4 could participate in the formation of different SEC subtypes by serving as a scaffolding protein. ${ }^{34}$ Other researchers also found that AFF4 binds to Tat-P-TEFb, which indirectly stimulates recognition of the HIV promoter. ${ }^{33}$ In addition, both AFFl and AFF4 are scaffolding proteins that link different parts of SECs. ${ }^{28,34}$ Despite these similarities, these two proteins have opposite effects on the 
osteogenic differentiation of MSCs. Overexpression of AFF 1 impairs MSC-mediated bone formation, whereas overexpression of AFF4 enhances this process. Mechanistically, ChIP experiments suggest that both AFFl and AFF4 could function as transcription factors to mediate the transcriptional activation of the key regulators in MSC osteogenic differentiation by binding to the promoter regions of these genes. AFF1, but not AFF4, affects differentiation by regulating DKK I transcription, while AFF4 regulates IDI transcription to control osteoblastic differentiation through the BMP pathway. These findings are consistent with a previous report that demonstrated that genes regulated by SECs containing AFFl or AFF4 are largely non-overlapping. ${ }^{43}$

On the basis on previous studies and the findings of this study, we speculate that there are several potential reasons for the different effects of AFFl and AFF4 on the osteoblastic differentiation of MSCs. It is well known that SECs regulate gene expression at the transcriptional level. ${ }^{38}$ Although AFFl and AFF4 are components of SECs, they may be independently localized and are not found together in a single SEC. ${ }^{27,43}$ These two proteins may also form a heterodimer, which is not necessary in SEC assembly. ${ }^{43} \mathrm{~A}$ previous study showed that the subnuclear distribution of AFFl is diffuse, while AFF4 is mainly found on SC35 in nuclear speckles. ${ }^{28}$ Furthermore, the gene targets of AFFl and AFF4 are largely distinct. ${ }^{43}$ Taken together, our data further demonstrated that these two proteins function differently during osteogenesis by regulating different signal pathways.

In summary, we show that AFF1 and AFF4 differentially regulate the osteogenic differentiation of human MSCs in vitro and MSC-mediated bone formation in vivo. Mechanically, AFF1 controls the transcription of DDK1, while AFF4 is required for IDI transcription and BMP2induced responses. Our data indicate that AFF1 and AFF4 are critical epigenetic regulators of the osteogenic differentiation of human MSCs.

\section{Acknowledgements}

This work was supported by grants from the National Natural Science Foundation of China (NSFC, 81722014, 81571001, 81500354, and 81621062), Sichuan Province Science and Technology Innovation Team Program (2017TD0016), and State Key Laboratory of Oral Diseases (SKLOD201704).

\section{Competing interests}

The authors declare no conflict of interest.

\section{References}

1 Bianco P, Robey PG, Simmons PJ. Mesenchymal stem cells: revisiting history, concepts, and assays. Cell Stem Cell 2008; 2: 313-319.

2 Moroni L, Fornasari PM. Human mesenchymal stem cells: a bank perspective on the isolation, characterization and potential of alternative sources for the regeneration of musculoskeletal tissues. J Cell Physiol 2013; 228: 680-687.

3 Campagnoli C, Roberts IA, Kumar S et al. Identification of mesenchymal stem/progenitor cells in human first-trimester fetal blood, liver, and bone marrow. Blood 2001; 98: 2396-2402.

4 Gronthos S, Franklin DM, Leddy HA et al. Surface protein characterization of human adipose tissue-derived stromal cells. J Cell Physiol 2001; 189: 54-63.

5 in't Anker PS, Noort WA, Scherjon SA et al. Mesenchymal stem cells in human second-trimester bone marrow, liver, lung, and spleen exhibit a similar immunophenotype but a heterogeneous multilineage differentiation potential. Haematologica 2003; 88: 845-852.

6 Miura M, Gronthos S, Zhao M et al. SHED: stem cells from human exfoliated deciduous teeth. Proc Natl Acad Sci USA 2003; 100: 5807-5812.

7 Raggatt LJ, Partridge NC. Cellular and molecular mechanisms of bone remodeling. J Biol Chem 2010; 285: 25103-25108.

8 Frith J, Genever P. Transcriptional control of mesenchymal stem cell differentiation. Transfus Med Hemother 2008; 35: 216-227.

9 Yuan $\mathrm{Q}$, Jiang $\mathrm{Y}$, Zhao $\mathrm{X}$ et al. Increased osteopontin contributes to inhibition of bone mineralization in FGF23-deficient mice. J Bone Miner Res 2014; 29: 693-704.

10 Yuan Q, Sato T, Densmore M et al. Deletion of PTH rescues skeletal abnormalities and high osteopontin levels in Klotho-/- mice. PLoS Genet 2012; 8: e1002726.

11 Kinoshita S, Kawai M. The FGF23/KLOTHO regulatory network and its roles in human disorders. Vitam Horm 2016; 101: 151-174.

12 Rahman MS, Akhtar N, Jamil HM et al. TGF-beta/BMP signaling and other molecular events: regulation of osteoblastogenesis and bone formation. Bone Res 2015; 3: 15005.

$13 \mathrm{Wu}$ M, Chen G, Li YP. TGF-beta and BMP signaling in osteoblast, skeletal development, and bone formation, homeostasis and disease. Bone Res 2016; 4: 16009.

14 Guo J, Liu M, Yang D et al. Suppression of Wnt signaling by Dkk1 attenuates PTH-mediated stromal cell response and new bone formation. Cell Metab 2010; 11: 161-171.

15 Song L, Liu M, Ono $\mathrm{N}$ et al. Loss of wnt/beta-catenin signaling causes cell fate shift of preosteoblasts from osteoblasts to adipocytes. J Bone Miner Res 2012; 27: 2344-2358.

16 Eslaminejad MB, Fani N, Shahhoseini M. Epigenetic regulation of osteogenic and chondrogenic differentiation of mesenchymal stem cells in culture. Cell J 2013; 15: 1-10.

17 Teven CM, Liu X, Hu N et al. Epigenetic regulation of mesenchymal stem cells: a focus on osteogenic and adipogenic differentiation. Stem Cells Int 2011; 2011: 201371.

18 Deng P, Chen QM, Hong C et al. Histone methyltransferases and demethylases: regulators in balancing osteogenic and adipogenic differentiation of mesenchymal stem cells. Int J Oral Sci 2015; 7: 197-204.

19 Robertson KD. DNA methylation and human disease. Nat Rev Genet 2005; 6: 597-610.

$20 \mathrm{Wu} \mathrm{H}$, Sun YE. Epigenetic regulation of stem cell differentiation. Pediatr Res 2006; 59: 21R-25R.

21 Lee HW, Suh JH, Kim AY et al. Histone deacetylase 1-mediated histone modification regulates osteoblast differentiation. Mol Endocrinol 2006; 20: 2432-2443.

22 Mueller D, Bach C, Zeisig D et al. A role for the MLL fusion partner ENL in transcriptional elongation and chromatin modification. Blood 2007; 110: $4445-4454$.

23 Bitoun E, Oliver PL, Davies KE. The mixed-lineage leukemia fusion partner AF4 stimulates RNA polymerase II transcriptional elongation 
and mediates coordinated chromatin remodeling. Hum Mol Genet 2007; 16: 92-106.

24 Hillman MA, Gecz J. Fragile XE-associated familial mental retardation protein 2 (FMR2) acts as a potent transcription activator. J Hum Genet 2001; 46: 251-259.

25 Bitoun E, Davies KE. The robotic mouse: unravelling the function of AF4 in the cerebellum. Cerebellum 2005; 4: 250-260.

26 He N, Zhou Q. New insights into the control of HIV-1 transcription: when Tat meets the 7SK snRNP and super elongation complex (SEC). J Neuroimmune Pharmacol 2011; 6: 260-268.

27 Biswas D, Milne TA, Basrur V et al. Function of leukemogenic mixed lineage leukemia 1 (MLL) fusion proteins through distinct partner protein complexes. Proc Natl Acad Sci USA 2011; 108: 15751-15756.

28 Melko M, Douguet D, Bensaid M et al. Functional characterization of the AFF (AF4/FMR2) family of RNA-binding proteins: insights into the molecular pathology of FRAXE intellectual disability. Hum Mol Genet 2011; 20: 1873-1885.

29 Domer PH, Fakharzadeh SS, Chen CS et al. Acute mixed-lineage leukemia $\mathrm{t}(4 ; 11)(\mathrm{q} 21 ; \mathrm{q} 23)$ generates an MLL-AF4 fusion product. Proc Natl Acad Sci USA 1993; 90: 7884-7888.

30 Taki T, Kano H, Taniwaki M et al. AF5q31, a newly identified AF4related gene, is fused to MLL in infant acute lymphoblastic leukemia with ins(5;11)(q31;q13q23). Proc Natl Acad Sci USA 1999; 96: 14535-14540.

31 Gecz J, Oostra BA, Hockey A et al. FMR2 expression in families with FRAXE mental retardation. Hum Mol Genet 1997; 6: 435-441.

32 Mak AB, Nixon AM, Moffat J. The mixed lineage leukemia (MLL) fusion-associated gene AF4 promotes CD133 transcription. Cancer Res 2012; 72: 1929-1934.

33 Schulze-Gahmen U, Lu H, Zhou Q et al. AFF4 binding to Tat-P-TEFb indirectly stimulates TAR recognition of super elongation complexes at the HIV promoter. eLife 2014; 3: $\mathrm{e} 02375$.

$34 \mathrm{Lu} \mathrm{H}, \mathrm{Li} \mathrm{Z}$, Xue Y et al. AFF1 is a ubiquitous P-TEFb partner to enable Tat extraction of P-TEFb from 7SK snRNP and formation of SECs for HIV transactivation. Proc Natl Acad Sci USA 2014; 111: E15-E24.
35 Liu W, Zhou L, Zhou C et al. GDF11 decreases bone mass by stimulating osteoclastogenesis and inhibiting osteoblast differentiation. Nat Commun 2016; 7: 12794.

36 Zhou C, Liu Y, Li X et al. DNA N6-methyladenine demethylase ALKBH1 enhances osteogenic differentiation of human MSCs. Bone Res 2016; 4: 16033.

37 Zou H, Zhao X, Sun $\mathrm{N}$ et al. Effect of chronic kidney disease on the healing of titanium implants. Bone 2013; 56: 410-415.

38 Luo Z, Lin C, Guest E et al. The super elongation complex family of RNA polymerase II elongation factors: gene target specificity and transcriptional output. Mol Cell Biol 2012; 32: 2608-2617.

39 Schneider VA, Mercola M. Wnt antagonism initiates cardiogenesis in Xenopus laevis. Genes Dev 2001; 15: 304-315.

40 Perk J, Iavarone A, Benezra R. Id family of helix-loop-helix proteins in cancer. Nat Rev Cancer 2005; 5: 603-614.

41 Ma C, Staudt LM. LAF-4 encodes a lymphoid nuclear protein with transactivation potential that is homologous to AF-4, the gene fused to MLL in $\mathrm{t}(4 ; 11)$ leukemias. Blood 1996; 87: 734-745.

42 von Bergh AR, Beverloo HB, Rombout P et al. LAF4, an AF4-related gene, is fused to MLL in infant acute lymphoblastic leukemia. Genes Chromosomes Cancer 2002; 35: 92-96.

$43 \mathrm{Lu} \mathrm{H}, \mathrm{Li} \mathrm{Z}$, Zhang W et al. Gene target specificity of the Super Elongation Complex (SEC) family: how HIV-1 Tat employs selected SEC members to activate viral transcription. Nucleic Acids Res 2015; 43: 5868-5879.

(a) This work is licensed under a Creative Commons Attribution 4.0 International License. The images or other third party material in this article are included in the article's Creative Commons license, unless indicated otherwise in the credit line; if the material is not included under the Creative Commons license, users will need to obtain permission from the license holder to reproduce the material. To view a copy of this license, visit http:/ / creativecommons.org/licenses/by/4.0/

(C) The Author(s) 2017 